\title{
Effects of Ectopic Pacing on Repolarization of the Chicken Left Ventricle
}

\author{
S. N. KHARIN, D. N. SHMAKOV, N. A. ANTONOVA \\ Laboratory of Cardiac Physiology, Institute of Physiology, Komi Science Centre of the Russian \\ Academy of Sciences, Syktyvkar, Komi Republic, Russia
}

Received January 25, 2008

Accepted May 21, 2008

On-line July 18, 2008

\section{Summary}

Effects of ectopic pacing on left ventricular repolarization were studied in six anesthetized open-chest chickens. In each animal, unipolar electrograms were acquired from as many as 98 sites with 14 plunge needles (seven transmural locations between epicardium and endocardium in each needle). Activation-recovery intervals (ARIs), corrected to the cycle length, were used for estimating repolarization. At baseline, the nonuniform ARI distribution in the left ventricle resulted in the apicobasal differences being greater than the transmural gradient. Nonuniform ARI prolongation caused by ectopic pacing resulted in decreasing the transmural repolarization gradient and increasing the differences in the apex-to-base direction. The basal, but not apical transmural differences contributed to the total left ventricular transmural gradient. The total left ventricular apicobasal gradient was contributed by the apicobasal differences in mid-myocardial and subendocardial layers more than in subepicardial ones. Thus, in in situ chicken hearts, the transmural and apicobasal ARI gradients exist within the left ventricle with the shortest ARIs in the basal subepicardium and the longest ARIs in the subendocardium of the apical and middle parts of the left ventricle. Apicobasal compared to transmural heterogeneity of local repolarization properties contributes more to the total left ventricular repolarization gradient.

\section{Key words}

Repolarization • Activation recovery interval • Heart ventricles • Pacing • Avian heart

\section{Corresponding author}

S. Kharin, Institute of Physiology of the Russian Academy of Sciences, 50 Pervomayskaya st., 167000, Syktyvkar, Komi Republic, Russia. Fax: +7(8212)240085.

E-mail: s.kharin@physiol.komisc.ru (s.kharin@mail.ru)

\section{Introduction}

At least three repolarization gradients exist in the heart ventricles of mammalian species: transmural (Higuchi and Nakaya 1984, Yan and Martin 2003, Hlaing et al. 2005), apicobasal (Nishimura et al. 1984, Cowan et al. 1988, Janse et al. 2005, Ramanathan et al. 2006, Azarov et al. 2007, 2008), and interventricular (Nishimura et al. 1984). However, several studies have demonstrated the absence of the former gradient in mammalian heart ventricles in vivo (Anyukhovsky et al. 1996, Taggart et al. 2001, Wang et al. 2002, Janse et al. 2005, Coronel et al. 2007, Zhang et al. 2007). Most likely, the ventricular repolarization gradients are differently expressed in the heart and are differently affected by various factors, e.g. electrolyte balance, temperature, ectopic excitation etc.

The use of different model animals contributes to understanding the evolution of a heart function and mechanisms of electrogenesis in the heart. In our investigations, birds were chosen as model animals. Vast majority of avian research on cardiac repolarization has centered only on the morphology of the $\mathrm{T}$ wave. Little is known about repolarization gradients in the heart ventricles in birds. Our recent studies have demonstrated apicobasal repolarization differences on the epicardial surface of the heart ventricles (Kharin 2004) and a transmural repolarization gradient in the left ventricular free wall (Kharin et al. 2007) at sinus rhythm in chickens. It is unknown which of the ventricular repolarization gradients (apicobasal or transmural) is more expressed in avian hearts. Effects of 
various factors on ventricular repolarization in birds have not been studied.

The objective of the present study is to evaluate the effects of ectopic pacing of the heart on left ventricular repolarization across and along the left ventricular free wall and to determine the contribution of apicobasal and transmural repolarization heterogeneity to the total left ventricular repolarization gradient in chickens.

\section{Methods}

\section{Animals and surgical procedure}

The study conforms with the Guide for the Care and Use of Laboratory Animals published by the US National Institutes of Health (NIH publication No 85-23, revised 1996). Six Brown laying hens Gallus gallus (weight 1.3-1.8 kg, 9-12 months old) were purchased from a poultry farm. Birds were anesthetized with an intramuscular injection of sodium thiopental $(120-150 \mathrm{mg} / \mathrm{kg})$ and placed in dorsal recumbency. After installation of artificial ventilation, the heart was exposed via bilateral thoracotomy. Throughout the experiment, the temperature of the avian body was in the range of $41-42{ }^{\circ} \mathrm{C}$. The heart was prevented from cooling and drying by warm saline $(0.85 \% \mathrm{NaCl})$. For this purpose, the heart was moistened by regular irrigation.

\section{Electrodes}

Unipolar electrograms were acquired from as many as 98 myocardial sites of the left ventricular free wall with plunge needles at sinus rhythm and under ectopic pacing. Fourteen plunge needles (diameter 0.35$0.40 \mathrm{~mm}$; length $5.0-7.5 \mathrm{~mm}$ ), each consisting of seven unipolar electrodes, were used. The diameter of each lead point was $0.07 \mathrm{~mm}$. A distance from centre to centre between electrode points was equal for each plunge needle $(0.65,0.80$ or $1 \mathrm{~mm})$. Thus, the distance from centre to centre between the first and seventh electrode points was 3.9, 4.8 or $6 \mathrm{~mm}$. Electrodes were made of copper insulated wires (diameter $0.07 \mathrm{~mm}$ ) fixed on a steel needle with an epoxy resin. Plunge needles were inserted into the myocardial wall according to one of schemes (Fig. 1). Figure 2 shows the original tracings of intramural electrograms.

Needle electrodes were placed through the skin of the medial part of each shoulder (the red electrode the right wing, the yellow electrode - the left wing) and through the skin of each thigh close to the knee joint (the green electrode - the left leg, the indifferent electrode the right leg) to obtain ECG recordings with the standard bipolar limb leads.

\section{Signal acquisition}

All unipolar electrograms and standard bipolar limb lead electrocardiograms were recorded simultaneously. The signals were isolated, amplified, multiplexed and recorded by a custom-designed 128-channel data acquisition system with the bandwidth of 0.05 to $1000 \mathrm{~Hz}$ at a sampling rate of $4000 \mathrm{~Hz}$ and an accuracy of 16 bits.

\section{Stimulation protocol}

Unipolar stimulation was used to generate ectopic beats of the heart ventricles. To obtain a stable state of ectopic excitation, the heart was driven for $30 \mathrm{~s}$. Hearts were paced with right-angled impulses of $3 \mathrm{~ms}$ duration at a frequency of $3 \mathrm{~Hz}$ (approximately 15-30\% higher than sinus rhythm). Amplitude of impulses was 3-7 V. The base and apex of the left ventricle were paced from epicardial and endocardial points.

\section{Data analysis}

To estimate local repolarization properties of myocardium, activation-recovery intervals (ARIs) were measured from unipolar electrograms as an interval between local depolarization and local repolarization times (Burgess et al. 1972). Local depolarization and repolarization times were defined as the minimum first derivative and the maximum first derivative of the unipolar electrogram in the period corresponding to the QRS complex and T wave in ECG, respectively. The computer-chosen (automatically) depolarization and repolarization times were reviewed and corrected if required. 322 electrograms of 588 ones $(6$ hens $\times$ 14 plunge needles $\times 7$ electrodes) were analyzed; remaining electrograms were unsatisfactory to analyze the T wave. All ARIs were corrected to the cardiac cycle length according to the Bazett's equation. Electrodes 1 and 2 of each plunge needle were considered to be in the subendocardial position, electrodes 3,4 and 5 in the midmyocardial position and electrodes 6 and 7 in the subepicardial position as stated in Tables 1 and 4 and in Figure 3.

Data are presented as means \pm SD. Statistical comparisons were carried out by paired and unpaired Student's $t$-test. 
(a)

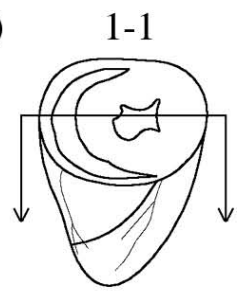

$1-1$

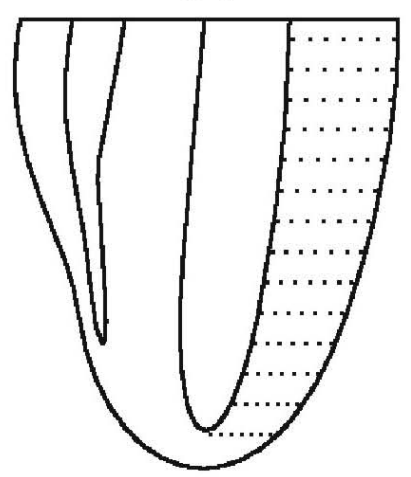

(b)

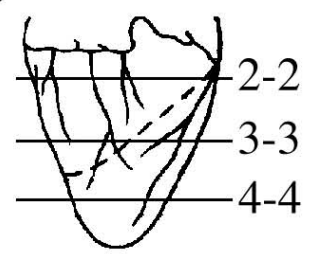

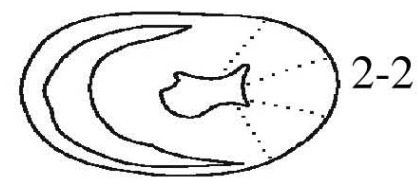

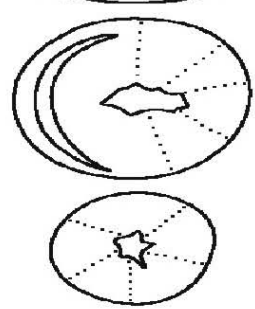

$3-3$

Fig. 1. The schemes $(\mathbf{a}, \mathbf{b})$ of insertion of plunge needles into the myocardial wall of the left ventricle. Sections of the heart ventricles: 1-1, longitudinal section; 2-2, 3-3, 4-4, transversal sections.

(a)

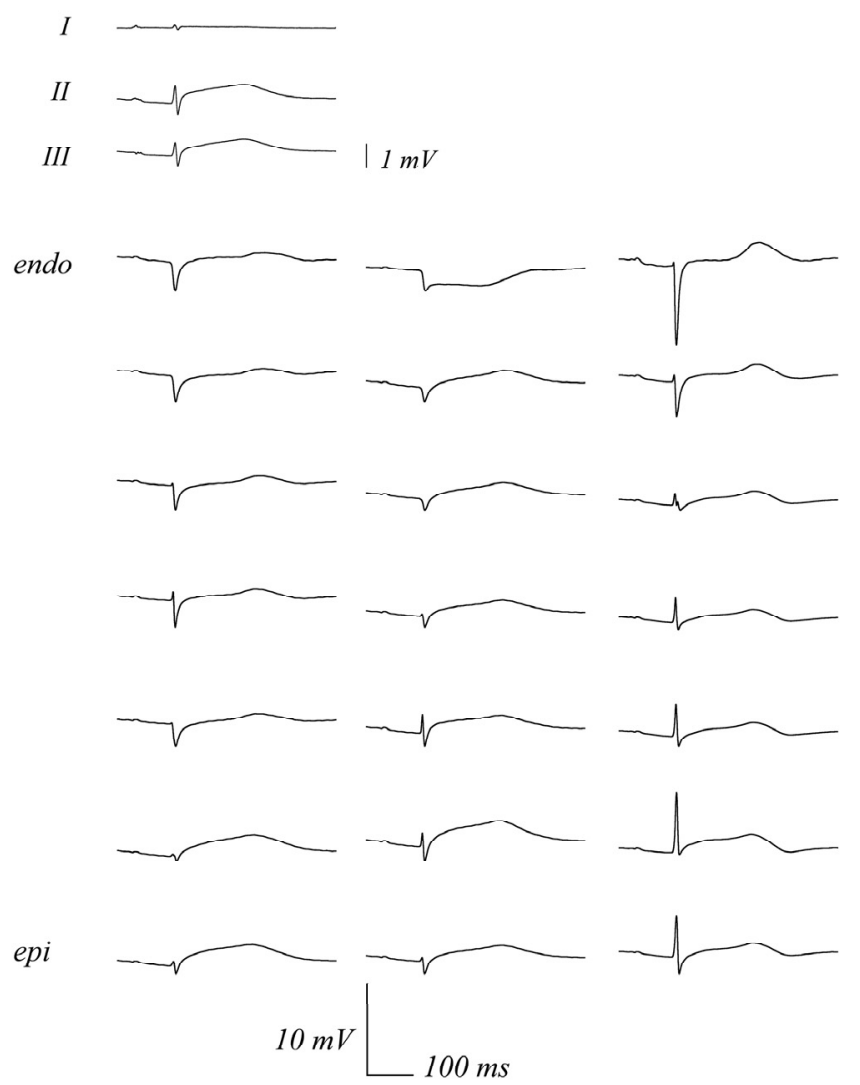

(b)
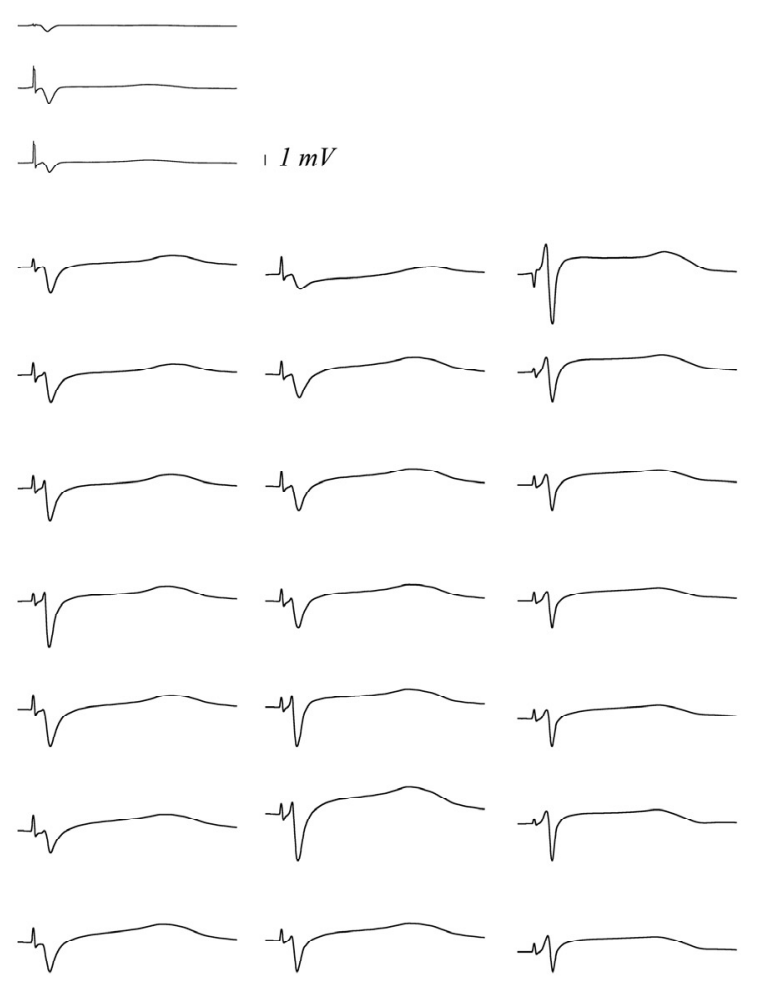

$10 \mathrm{mV} L 100 \mathrm{~ms}$

Fig. 2. Examples of electrograms recorded with three plunge needles within the chicken left ventricular wall, and ECG in the standard bipolar limb leads under sinus rhythm (a) and endocardial pacing of the left ventricular base (b). I, II and III, ECG in the standard bipolar limb leads; endo, endocardium; epi, epicardium.

\section{Results}

ARI distribution within the chicken left ventricular free wall at sinus rhythm

The pooled ARI of the whole left ventricular free wall was $257 \pm 31 \mathrm{~ms}$ at sinus rhythm, which was $150 \pm 14$ beats per minute (Table 1, “Average”).

\section{Apicobasal differences in ARIs}

ARIs in the basal third of the left ventricular free wall were the shortest, whereas ARIs in the middle third were the longest (Table 2). ARIs in the apical third of the left ventricular free wall were intermediate but closer to the latter. The apicobasal ARI distribution in each of three layers (subendocardial, mid-myocardial, 

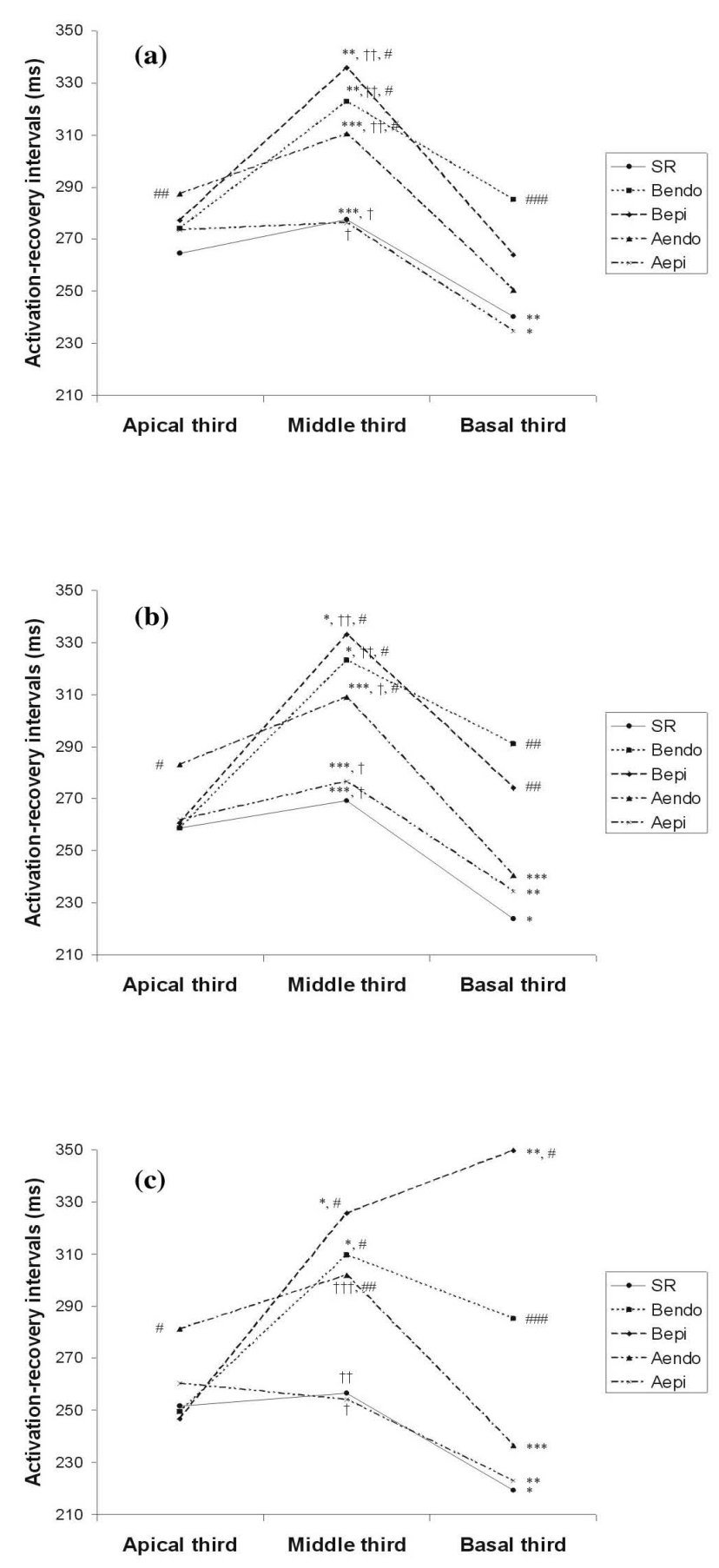

Fig. 3. Apicobasal differences in activation-recovery intervals in the subendocardial (a), mid-myocardial (b) and subepicardial (c) layers of the left ventricular free wall in all animals under sinus rhythm and ectopic pacing of the left ventricle. Two, three and two electrodes were considered to be at subepicardial, midmyocardial and subendocardial positions, respectively, in each seven-electrode plunge needle. SR, sinus rhythm; Bendo, endocardial pacing of the left ventricular base; Bepi, epicardial pacing of the left ventricular base; Aendo, endocardial pacing of the left ventricular apex; Aepi, epicardial pacing of the left ventricular apex. ${ }^{*} P<0.001,{ }^{* *} P<0.01,{ }^{* * *} P<0.05$ the middle / basal third vs. the apical one; ${ }^{\dagger} P<0.001,{ }^{+\dagger} P<0.01,{ }^{++\dagger} P<0.05$ the middle third vs. the basal one; \# $P<0.001$, \# $P<0.01$, \#\#\# $P<0.05$ vs. sinus rhythm. subepicardial) of the left ventricular wall coincided with the total apicobasal distribution in the left ventricular wall (Fig. 3, Table 2). The difference between ARIs in the apical and middle thirds of the left ventricular free wall was negligible in the subepicardial layer (252 \pm 24 vs. $257 \pm 29 \mathrm{~ms}$ ), whereas it was slightly greater in the midmyocardial (259 \pm 24 vs. $269 \pm 23 \mathrm{~ms}$, respectively) and subendocardial $(265 \pm 23$ vs. $277 \pm 19 \mathrm{~ms})$ layers. The difference between ARIs in the middle and basal thirds of the left ventricular free wall was the greatest in the midmyocardial layer ( $269 \pm 23$ vs. $224 \pm 48 \mathrm{~ms}$ ), whereas it was smaller in the subepicardial ( $257 \pm 29$ vs. $219 \pm 48 \mathrm{~ms})$ and subendocardial ( $277 \pm 19$ vs. $240 \pm 43 \mathrm{~ms})$ layers. The latter was similar to the difference between the apical and basal ARIs in the mid-myocardial (35 ms) and subepicardial (33 ms) layers, which was more than that in the subendocardial layer (25 ms).

\section{Transmural differences in ARIs}

ARIs in the subepicardial layers were shorter than in the subendocardial ones (Table 1). The lengthening of ARIs from the subepicardium to the subendocardium was smooth and even in some regions of the ventricular wall, whereas it was sharp in the others. However, in some regions of the left ventricular free wall, ARIs were shorter in the subendocardial than in subepicardial layers. As a result, the transmural gradient of ARIs (the difference between ARIs recorded from the subepicardial and subendocardial layers of myocardium) had a large variation and was in the average $16 \pm 14 \mathrm{~ms}$ (Table 3).

ARI prolongation across the left ventricular free wall was more significant in the middle and apical thirds than in the basal third (Table 4). In all thirds of the ventricular wall, the subepicardial ARIs were, as a rule, shorter than the subendocardial ones. As a result, the direction of the transmural gradient of ARIs in each third of the ventricular wall coincided with the direction of the total transmural gradient in the left ventricular wall (Table 3).

\section{Effect of ectopic pacing on the pooled ARI}

The pooled ARI was significantly prolonged under ectopic pacing (Table 1, "Average"). Across the ventricular wall, this prolongation resulted mainly from the lengthening of the subepicardial and mid-myocardial ARIs, but not the subendocardial ones (Table 1). Prolongation of the pooled ARI along the ventricular wall was mostly due to the lengthening of ARIs in the basal and middle thirds of the ventricular wall under basal pacing and in the middle 
Table 1. Activation-recovery intervals in seven myocardial layers of the left ventricular free wall in all animals under sinus rhythm and ectopic pacing of the left ventricle.

\begin{tabular}{|c|c|c|c|c|c|}
\hline \multirow[t]{2}{*}{$\begin{array}{l}\text { Locations of } \\
\text { electrodes }\end{array}$} & \multirow[t]{2}{*}{$\begin{array}{l}\text { Sinus } \\
\text { rhythm }\end{array}$} & \multicolumn{4}{|c|}{ of the base } \\
\hline & & endocardial & epicardial & endocardial & epicardial \\
\hline 1 (endocardium) & $270 \pm 28^{* *}$ & $291 \pm 47^{\# \#}$ & $295 \pm 54^{\#}$ & $292 \pm 49^{* * *, \#}$ & $273 \pm 29^{* * *}$ \\
\hline 2 & $261 \pm 28^{* *}$ & $283 \pm 49$ \#\# & $286 \pm 53^{* * * * \#}$ & $291 \pm 55^{\#}$ & $265 \pm 28$ \\
\hline 3 & $260 \pm 30^{* *}$ & $284 \pm 48^{\#}$ & $283 \pm 55^{\#}$ & $287 \pm 56^{\#}$ & $263 \pm 29$ \\
\hline 4 & $256 \pm 32^{* *}$ & $284 \pm 57^{\#}$ & $287 \pm 62^{\#}$ & $291 \pm 57^{*}, \#$ & $263 \pm 26$ \\
\hline 5 & $254 \pm 32^{*}$ & $277 \pm 53^{* * *}, \#$ & $284 \pm 57^{\#}$ & $284 \pm 57^{\#}$ & $260 \pm 37$ \\
\hline 6 & $250 \pm 32^{* *}$ & $274 \pm 54^{* *}, \# \#$ & $287 \pm 59$ \# & $283 \pm 57^{\#}$ & $253 \pm 31^{*}$ \\
\hline 7 (epicardium) & $247 \pm 30^{\dagger}$ & $268 \pm 53^{\# \#}$ & $274 \pm 57^{\#}$ & $281 \pm 58^{\dagger, \#}$ & $255 \pm 32^{\dagger}$ \\
\hline Average & $257 \pm 31$ & $280 \pm 51^{\#}$ & $285 \pm 56^{\#}$ & $287 \pm 55^{\#}$ & $262 \pm 31$ \# \\
\hline
\end{tabular}

Data are mean \pm S.D. ${ }^{*} P<0.001,{ }^{* *} P<0.01,{ }^{* * *} P<0.05$ vs. the below value; ${ }^{\dagger} P<0.001$ epicardium vs. endocardium; ${ }^{\#} P<0.001,{ }^{\# \#}$ $P<0.01$ vs. sinus rhythm.

Table 2. Apicobasal differences in activation-recovery intervals in the left ventricular free wall in all animals under sinus rhythm and ectopic pacing of the left ventricle.

\begin{tabular}{|c|c|c|c|c|c|}
\hline \multirow[t]{2}{*}{$\begin{array}{l}\text { The part of the left } \\
\text { ventricular wall }\end{array}$} & \multirow[t]{2}{*}{$\begin{array}{l}\text { Sinus } \\
\text { rhythm }\end{array}$} & \multicolumn{4}{|c|}{ of the base } \\
\hline & & endocardial & epicardial & endocardial & epicardial \\
\hline Basal third & $228 \pm 47$ & $286 \pm 45^{\#}$ & $290 \pm 60^{\#}$ & $244 \pm 15$ & $231 \pm 20$ \\
\hline Middle third & $267 \pm 24^{*}, \dagger$ & $319 \pm 22^{*}, \dagger, \#$ & $331 \pm 22^{*}, \dagger, \#$ & $307 \pm 52^{*}, \dagger, \#$ & $272 \pm 24$ * \\
\hline Apical third & $258 \pm 24^{\S}$ & $260 \pm 51 \S \S \S$ & $262 \pm 53 \S \S \S$ & $284 \pm 56^{\#, \S \S}$ & $264 \pm 31^{\S}$ \\
\hline
\end{tabular}

Data are mean \pm S.D. ${ }^{*} P<0.001,{ }^{* *} P<0.01,{ }^{* * *} P<0.05$ the middle part vs. the basal one; ${ }^{\dagger} P<0.001,{ }^{\text {t+ }} P<0.01,{ }^{\dagger+\dagger} P<0.05$ the middle part vs. the apical one; ${ }^{\#} P<0.001$, ${ }^{\# \#} P<0.01$, \#\# $P<0.05$ vs. sinus rhythm; ${ }^{\S} P<0.001,{ }^{\S \S} P<0.01,{ }^{\S \S \S} P<0.05$ the apical third vs. the basal one.

Table 3. Differences between activation-recovery intervals recorded from the subepicardial and subendocardial layers of the myocardium in the left ventricular free wall in all animals under sinus rhythm and ectopic pacing of the left ventricle.

\begin{tabular}{llllll}
\hline $\begin{array}{l}\text { The part of the left } \\
\text { ventricular wall }\end{array}$ & $\begin{array}{l}\text { Sinus } \\
\text { rhythm }\end{array}$ & \multicolumn{2}{c}{$\begin{array}{c}\text { Pacing } \\
\text { of the base } \\
\end{array}$} & & \multicolumn{2}{c}{$\begin{array}{c}\text { endocardial } \\
\text { epicardial }\end{array}$} & \multicolumn{2}{c}{$\begin{array}{c}\text { of the apex } \\
\text { endocardial }\end{array}$} & epicardial \\
\hline $\begin{array}{l}\text { Total wall } \\
\text { Basal third }\end{array}$ & $16 \pm 14$ & $11 \pm 28$ & $3 \pm 24^{*}$ & $9 \pm 16^{* *}$ & $13 \pm 19$ \\
Middle third & $22 \pm 24$ & $-1 \pm 70$ & $-45 \pm 42^{* *}$ & $3 \pm 9$ & $6 \pm 35$ \\
Apical third & $23 \pm 12$ & $15 \pm 22$ & $10 \pm 15^{* *}$ & $9 \pm 25^{* *}$ & $20 \pm 11$ \\
& $11 \pm 10$ & $11 \pm 18$ & $9 \pm 15^{* *}$ & $10 \pm 12$ & $13 \pm 17$ \\
\hline
\end{tabular}

Data are mean \pm S.D. ${ }^{*} P<0.01,{ }^{* *} P<0.05$ vs. sinus rhythm.

and apical thirds under apical pacing (Table 2). ARI prolongation was greater along than across the left ventricular wall. Epicardial pacing of the left ventricular apex resulted in ARI prolongation to a lesser degree compared with other pacing sites (Tables 1 and 2).
Effect of ectopic pacing on transmural differences in ARI Under ectopic pacing, except of epicardial pacing of the left ventricular apex, ARIs were significantly prolonged in all myocardial layers (Table 1). This prolongation was nonuniform across the ventricular wall. As a rule, the subepicardial ARIs were more 
Table 4. Activation-recovery intervals in seven myocardial layers in each of the apical, middle and basal thirds of the left ventricular free wall in all animals under sinus rhythm and ectopic pacing of the left ventricle.

\begin{tabular}{|c|c|c|c|c|c|}
\hline \multirow[t]{2}{*}{$\begin{array}{l}\text { Locations of } \\
\text { electrodes }\end{array}$} & \multirow[t]{2}{*}{$\begin{array}{l}\text { Sinus } \\
\text { rhythm }\end{array}$} & \multicolumn{4}{|c|}{ of the apex } \\
\hline & & endocardial & epicardial & endocardial & epicardial \\
\hline \multicolumn{6}{|c|}{ The basal third of the left ventricular free wall } \\
\hline 1 (endocardium) & $246 \pm 41$ & $283 \pm 29$ & $276 \pm 58$ & $259 \pm 20$ & $236 \pm 35$ \\
\hline 2 & $233 \pm 50$ & $288 \pm 40$ & $240 \pm 10$ & $242 \pm 10$ & $234 \pm 26$ \\
\hline 3 & $227 \pm 55$ & $291 \pm 41$ & $244 \pm 40$ & $243 \pm 19$ & $234 \pm 25$ \\
\hline 4 & $223 \pm 49$ & $294 \pm 44$ & $285 \pm 86$ & $249 \pm 7$ & $237 \pm 12$ \\
\hline 5 & $222 \pm 50^{* *}$ & $289 \pm 66^{* * *}$ & $272 \pm 67$ & $232 \pm 4$ & $234 \pm 10$ \\
\hline 6 & $217 \pm 51$ & $286 \pm 65$ & $350 \pm 39^{\# \# \#}$ & $235 \pm 10$ & $225 \pm 10^{\# \# \# ~}$ \\
\hline 7 (epicardium) & $221 \pm 50$ & $285 \pm 68$ & $350 \pm 39^{\# \# \#}$ & $238 \pm 6$ & $220 \pm 33$ \\
\hline
\end{tabular}

\begin{tabular}{|c|c|c|c|c|c|}
\hline \multicolumn{6}{|c|}{ The middle third of the left ventricular free wall } \\
\hline 1 (endocardium) & $282 \pm 22^{* * *}$ & $323 \pm 16^{\#}$ & $337 \pm 23^{\#}$ & $313 \pm 47^{\# \# \#}$ & $280 \pm 21$ \\
\hline 2 & $272 \pm 16^{* * *}$ & $322 \pm 5^{* * *}, \# \#$ & $333 \pm 13^{\#}$ & $308 \pm 50 \#$ & $273 \pm 17$ \\
\hline 3 & $271 \pm 21$ & $321 \pm 14^{\#}$ & $333 \pm 20^{\#}$ & $305 \pm 52$ & $275 \pm 19$ \\
\hline 4 & $270 \pm 24^{* * *}$ & $332 \pm 28$ \#\# & $336 \pm 19^{\#}$ & $316 \pm 50$ \#\#\# & $278 \pm 30$ \\
\hline 5 & $266 \pm 25^{* * *}$ & $315 \pm 20^{\# \#}$ & $330 \pm 26^{\#}$ & 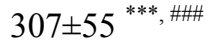 & $277 \pm 36^{* * *}$ \\
\hline 6 & $260 \pm 27$ & $314 \pm 222^{\#}$ & $328 \pm 27^{\#}$ & $303 \pm 57^{\# \# \#}$ & $258 \pm 10^{* * *}$ \\
\hline 7 (epicardium) & $253 \pm 30^{\dagger}$ & $305 \pm 35^{\# \# \#}$ & $323 \pm 29$ ††, \#\# & $300 \pm 51$ & $251 \pm 10^{\dagger}$ \\
\hline \multicolumn{6}{|c|}{ The apical third of the left ventricular free wall } \\
\hline 1 (endocardium) & $268 \pm 24^{* * *}$ & $279 \pm 54^{* * *}$ & $279 \pm 56$ & $284 \pm 52$ & $276 \pm 27$ \\
\hline 2 & $262 \pm 22$ & $271 \pm 52$ & $276 \pm 55$ & $290 \pm 56$ **,\#\# & $271 \pm 26^{* * *,}$,\#\# \\
\hline 3 & $261 \pm 22^{* *}$ & $265 \pm 49$ & $262 \pm 53$ & $284 \pm 58 \ldots \#$ & $266 \pm 29 \# \#$ \\
\hline 4 & $258 \pm 24$ & $257 \pm 54$ & $258 \pm 56$ & $285 \pm 59^{* *}, \# \#$ & $259 \pm 22 \ldots$ \\
\hline 5 & $257 \pm 26^{* * *}$ & $255 \pm 53$ & $262 \pm 56$ & $281 \pm 57^{\# \# \#}$ & $259 \pm 39$ \\
\hline 6 & $254 \pm 25^{*}$ & $249 \pm 52$ & $250 \pm 50$ & $281 \pm 58^{* * *}, \ldots \#$ & $260 \pm 34$ \\
\hline 7 (epicardium) & $249 \pm 24^{\dagger}$ & $249 \pm 51^{\dagger \dagger}$ & $243 \pm 49$ & $282 \pm 59{ }^{\dagger \dagger, \# \# ~}$ & $260 \pm 34^{\dagger,}$ \#\# \\
\hline
\end{tabular}

Data are mean \pm S.D. ${ }^{*} P<0.001,{ }^{* *} P<0.01,{ }^{* * *} P<0.05$ vs. the below value; ${ }^{\dagger} P<0.001,{ }^{+\dagger} P<0.01,{ }^{++\dagger} P<0.05$ epicardium vs. endocardium; " $P<0.001, \#$ \# $P<0.01, \# \# P<0.05$ vs. sinus rhythm.

prolonged than the subendocardial ones that resulted in a decrease of the total transmural gradient of ARIs in the left ventricular wall (Table 3).

Compared with baseline, ARI prolongation across the left ventricular free wall was significant mainly in the middle and apical thirds under basal and apical pacing, respectively (Table 4). Although there was nonuniform prolongation of the subepicardial and subendocardial ARIs under ectopic pacing, the subepicardial ARIs were, as a rule, shorter than the subendocardial ones in all thirds of the ventricular wall, except of the basal third under basal pacing (Table 4). As a result, the transmural gradient was decreased in the basal and middle thirds, but not in the apical third of the left ventricular free wall (Table 3 ). The decrease of the transmural gradient was more expressed in the basal third of the ventricular wall than in the middle one. Besides, the transmural gradient in the basal third was inverted under basal pacing of the left ventricle.

Effect of ectopic pacing on apicobasal differences in ARIs Under ectopic pacing, ARIs in all three layers of the left ventricular wall were prolonged most of all in the middle third of the ventricular wall (Fig. 3). In comparison with each other, a degree of prolongation of the basal and apical ARIs depended on a pacing site. In 
all three layers, ectopic pacing from the left ventricular base lengthened the basal ARIs with the apical ARIs being not or insignificantly prolonged. The contrary situation was observed for ARIs in all three layers under endocardial pacing of the left ventricular apex. ARI prolongation under epicardial pacing of the left ventricular apex was more complex and insignificant.

The differences in ARIs between the middle and basal thirds of the left ventricular wall were changed insignificantly except under endocardial pacing of the left ventricular apex Table 2). In contrast, the ARI differences between the middle and apical thirds of the left ventricular wall were increased more than twofold, except of under apical epicardial pacing. At the same time, the ARI differences between the apical and basal thirds of the left ventricular wall changed insignificantly.

Under endocardial ectopic pacing of both the apex and base of the left ventricle, the apicobasal gradient in each layer of the left ventricular wall (Fig. 3) coincided with the total apicobasal gradient in the left ventricular wall (Table 2). In contrast, under subepicardial pacing of the left ventricular apex, the apicobasal gradient in the subendocardial and mid-myocardial layers of the left ventricular wall coincided with the total apicobasal gradient in the left ventricular wall. Only the apicobasal gradient in the mid-myocardial layer of the left ventricular wall corresponded to the total apicobasal gradient in the left ventricular wall under subepicardial pacing of the left ventricular base.

\section{Discussion}

The major findings of the present study on chicken heart are as follows: 1) at baseline, the nonuniform ARI distribution in the left ventricle resulted in the apicobasal gradient being greater than the transmural gradient, 2) Non-uniform ARI prolongation caused by ectopic pacing resulted in a decreasing of the transmural repolarization gradient and increasing of the differences in the apex-to-base direction, 3) the basal, but not apical transmural differences contributed to the total left ventricular transmural gradient, and 4) the total left ventricular apicobasal gradient was contributed by the apicobasal differences in mid-myocardial and subendocardial layers more than by those in subepicardial ones.

Investigations of repolarization describe either only transmural or apicobasal differences in local repolarization durations, action potential durations or monophasic action potential durations. Majority of the above investigations were made on ventricular preparations or isolated myocytes, but not in vivo hearts. Our study describes both the transmural and apicobasal gradients of ARIs in the in situ chicken left ventricle.

No differences in repolarization among myocardial layers were shown in in vivo intact normal hearts of dogs (Anyukhovsky et al. 1996, Janse et al. 2005, Coronel et al. 2007), humans (Taggart et al. 2001), sheep (Wang et al. 2002, Zhang et al. 2007). The transmural repolarization gradient in the chicken left ventricle in situ was such that ARIs in the subepicardial layer were shorter than in the subendocardial one. These results are consistent with observations of action potential durations (Main et al. 1998, Xu et al. 2001, Aiba et al. 2003, Wan et al. 2003, Idriss and Wolf 2004) and monophasic action potential durations (Liu et al. 2003) in mammals and with the ARI distribution in chicken hearts (Kharin et al. 2007). In hens, the basal transmural differences contributed to the total left ventricular transmural gradient more than apical ones.

$M$ cells contributing to transmural heterogeneity of action potential durations, have been detected by microelectrode techniques in canine (Sicouri and Antzelevitch 1991), human (Drouin et al. 1995), guinea pig (Sicouri et al. 1996), porcine (Stankovičová et al. 2000), rabbit (Li et al. 2002), murine (Liu et al. 2003) and feline (Aiba et al. 2003) left ventricular myocardium. The above descriptions of $\mathrm{M}$ cells have been made on ventricular preparations, myocardial strips or enzymatically isolated myocytes. At the same time, a distinct layer of $\mathrm{M}$ cells may not be observed in the left ventricle of smaller animal species (Hlaing et al. 2005). In vivo results have shown no differences in repolarization among myocardial layers in intact normal hearts of dogs (Anyukhovsky et al. 1996, Janse et al. 2005, Coronel et al. 2007), humans (Taggart et al. 2001) or sheep (Wang et al. 2002, Zhang et al. 2007). At present, there are no evidences for the existence of $\mathrm{M}$ cells in the avian left ventricle. This question remains to be answered. $M$ cells are absent in the chicken left ventricle or are masked by intercellular coupling (Conrath et al. 2004). Our previous study (Kharin et al. 2007) and the present results do not demonstrate the long ARIs in the mid-myocardial region of in vivo chicken hearts at baseline and during ectopic pacing. The transmural ARI distribution in the normal chicken left ventricle is a smooth continuum. 
In mammals, action potential durations or ARIs were demonstrated to be shorter in the apex compared with the base (Nishimura et al. 1984, Laurita et al. 1996, Baker et al. 2000, Choi and Salama 2000, Szentadrassy et al. 2005, Mantravadi et al. 2007, Ramanathan et al. 2006, Azarov et al. 2007). In other investigations, the longer repolarization was found in the apex compared with the base (Toyoshima et al. 1981, Cowan et al. 1988, Cheng et al. 1999, Janse et al. 2005). The present results are consistent with both above observations. The differences in the apex-to-base direction in the chicken left ventricle were such that the apical ARIs were longer than the basal ones and shorter than ARIs in the middle part of the left ventricle, and the latter were the longest. Besides, the apicobasal differences in the subendocardial and mid-myocardial layers contributed to the total left ventricular apicobasal gradient more than in the subepicardial layers. There is some discrepancy with our recent investigation, concerning the ARI distribution on the chicken ventricular epicardium with ARIs increasing progressively from the apex to the base (Kharin 2004). This discrepancy can be related to regional heterogeneity in expression of ionic currents throughout ventricular myocardium (Bryant et al. 1998, Main et al. 1998, Cheng et al. 1999, Xu et al. 2001, Szabó et al. 2005, Szentadrassy et al. 2005) and in their sensitivity to different factors, such as ionic concentrations (Wan et al. 2000), hormones (Daleau and Turgeon 1994, Wang et al. 1999), adrenoceptor stimulation (Bosch et al. 2002, Volders et al. 2003, Rocchetti et al. 2006), the autonomic nervous system tone (Tatewaki et al. 2003, Conrath and Opthof 2006) and spatial heterogeneity of effects of the autonomic nervous system on ventricular repolarization (Mantravadi et al. 2007). In addition, the difference in the ARI distributions might be related to a dissimilar dependence of ARIs on heart rate in different ventricular parts. In the present study, heart rate was $150 \pm 14$ beats per minute, whereas it was $225 \pm 20$ beats per minute in the previous investigation (Kharin 2004).

Unexpectedly, the ARIs at sinus rhythm and under epicardial pacing of the left ventricular apex were rather close (Fig. 3). An additional analysis is needed to explain this observation. However, we suppose that it might be related to dependence of the ARI distribution and repolarization sequence on the activation sequence.
On the whole, ARI prolongation in the chicken left ventricle under ectopic pacing is in agreement with action potential prolongation in chronically paced mammalian hearts (Kääb et al. 1996, Tsuji et al. 2000), and with action potential prolongation following a period of the altered activation sequence in wedge preparations of canine ventricles (Libbus and Rosenbaum 2003). Our results indicate that the apicobasal repolarization differences are greater than the transmural ones at both baseline and ectopic pacing. Under ectopic pacing, ARI prolongation is greater in the apex-to-base compared to transmural direction. Both our observations support the suggestion that apicobasal heterogeneity in local repolarization properties contributes to the total left ventricular repolarization gradient more than transmural heterogeneity.

In conclusion, in the in situ chicken heart at sinus rhythm, the transmural and apicobasal gradients of ARIs exist within the left ventricular free wall with the shortest ARIs in the basal subepicardium and the longest ARIs in the subendocardium of the apical and middle parts of the left ventricle. This study provides data in support of predominance of apicobasal compared to transmural heterogeneity of local repolarization properties, and of a greater contribution of the apicobasal gradient to the total left ventricular repolarization gradient compared with the transmural gradient. A correlation between the changes in the repolarization gradients and changes in the electrocardiographic $\mathrm{T}$ wave during ectopic pacing of the avian heart ventricles is of certain interest for the genesis of the $\mathrm{T}$ wave. It was no the object of the present study. The effects of the changes in the repolarization gradients on the $\mathrm{T}$ wave in birds remain to be elucidated.

\section{Conflict of Interest}

There is no conflict of interest.

\section{Acknowledgements}

This study was supported by the Russian Foundation for Basic Research (grant No 06-04-48022), the Russian Science Support Foundation, and the Ural Branch of the Russian Academy of Sciences (the program of support for basic research performed in the Ural Branch of the Russian Academy of Sciences in association with the Far Eastern Branch of the Russian Academy of Sciences). 


\section{References}

AIBA T, SHIMIZU W, INAGAKI M, HIDAKA I, TATEWAKI T, SUNAGAWA K: Transmural heterogeneity of the action potential configuration in the feline left ventricle. Circ J 67: 449-454, 2003.

ANYUKHOVSKY EP, SOSUNOV EA, ROSEN MR: Regional differences in electrophysiological properties of epicardium, midmyocardium, and endocardium. In vitro and in vivo correlations. Circulation 94: 1981-1988, 1996.

AZAROV JE, SHMAKOV DN, VITYAZEV VA, ROSHCHEVSKAYA IM, ROSHCHEVSKY MP: Activation and repolarization patterns in the ventricular epicardium under sinus rhythm in frog and rabbit hearts. Comp Biochem Physiol A Mol Integr Physiol 146: 310-316, 2007.

AZAROV JE, SHMAKOV DN, VITYAZEV VA, ROSHCHEVSKAYA IM, ARTEYEVA NV, KHARIN SN, ROSHCHEVSKY MP: Ventricular repolarization pattern under heart cooling in the rabbit. Acta Physiol (Oxf) 193: $129-138,2008$.

BAKER LC, LONDON B, CHOI BR, KOREN G, SALAMA G: Enhanced dispersion of repolarization and refractoriness in transgenic mouse hearts promotes reentrant ventricular tachycardia. Circ Res 86: 396-407, 2000.

BOSCH RF, SCHNECK AC, KIEHN J, ZHANG W, HAMBROCK A, EIGENBERGER BW, RÜB N, GOGEL J, MEWIS C, SEIPEL L, KÜHLKAMP V: $\beta_{3}$-Adrenergic regulation of an ion channel in the heart-inhibition of the slow delayed rectifier potassium current $\mathrm{I}_{\mathrm{Ks}}$ in guinea pig ventricular myocytes. Cardiovasc Res 56: 393403, 2002.

BRYANT SM, WAN X, SHIPSEY SJ, HART G: Regional differences in the delayed rectifier current (IKr and IKs) contribute to the differences in action potential duration in basal left ventricular myocytes in guinea-pig. Cardiovasc Res 40: 322-331, 1998.

BURGESS MJ, GREEN LS, MILLAR K, WYATT R, ABILDSKOV JA: The sequence of normal ventricular recovery. Am Heart $J$ 84: 660-669, 1972.

CHENG J, KAMIYA K, LIU W, TSUJI Y, TOYAMA J, KODAMA I: Heterogeneous distribution of the two components of delayed rectifier $\mathrm{K}^{+}$current: a potential mechanism of the proarrhythmic effects of methanesulfonanilideclass III agents. Cardiovasc Res 43: 135-147, 1999.

CHOI BR, SALAMA G: Simultaneous maps of optical action potentials and calcium transients in guinea-pig hearts: mechanisms underlying concordant alternans. J Physiol Lond 529: 171-188, 2000.

CONRATH CE, OPTHOF T: Ventricular repolarization: an overview of (patho)physiology, sympathetic effects and genetic aspects. Prog Biophys Mol Biol 92: 269-307, 2006.

CONRATH CE, WILDERS R, CORONEL R, DE BAKKER JM, TAGGART P, DE GROOT JR, OPTHOF T: Intercellular coupling through gap junctions masks $\mathrm{M}$ cells in the human heart. Cardiovasc Res 62: 407-414, 2004.

CORONEL R, OPTHOF T, PLOTNIKOV AN, WILMS-SCHOPMAN FJ, SHLAPAKOVA IN, DANILO P JR, SOSUNOV EA, ANYUKHOVSKY EP, JANSE MJ, ROSEN MR: Long-term cardiac memory in canine heart is associated with the evolution of a transmural repolarization gradient. Cardiovasc Res 74: 416-425, 2007.

COWAN JC, HILTON CJ, GRIFFITHS CJ, TANSUPHASWADIKUL S, BOURKE JP, MURRAY A, CAMPBELL RW: Sequence of epicardial repolarisation and configuration of the T wave. Br Heart J 60: 424-433, 1988.

DALEAU P, TURGEON J: Angiotensin II modulates the delayed rectifier potassium current of guinea pig ventricular myocytes. Pflugers Arch 427: 553-555, 1994.

DROUIN E, CHARPENTIER F, GAUTHIER C, LAURENT K, LE MAREC H: Electrophysiologic characteristics of cells spanning the left ventricular wall of human heart: evidence for presence of M cells. J Am Coll Cardiol 26: 185-192, 1995.

HIGUCHI T, NAKAYA Y: T wave polarity related to the repolarization process of epicardial and endocardial ventricular surfaces. Am Heart J 108: 290-295, 1984.

HLAING T, DIMINO T, KOWEY PR, YAN GX: ECG repolarization waves: their genesis and clinical implications. Ann Noninv Electrocardiol 10: 211-223, 2005. 
IDRISS SF, WOLF PD: Transmural action potential repolarization heterogeneity develops postnatally in the rabbit. J Cardiovasc Electrophysiol 15: 795-801, 2004.

JANSE MJ, SOSUNOV EA, CORONEL R, OPTHOF T, ANYUKHOVSKY EP, DE BAKKER JM, PLOTNIKOV AN, SHLAPAKOVA IN, DANILO P JR, TIJSSEN JG, ROSEN MR: Repolarization gradients in the canine left ventricle before and after induction of short-term cardiac memory. Circulation 112: 1711-1718, 2005.

KÄÄB S, NUSS HB, CHIAMVIMONVAT N, O'ROURKE B, PAK PH, KASS DA, MARBAN E, TOMASELLI GF: Ionic mechanism of action potential prolongation in ventricular myocytes from dogs with pacing-induced heart failure. Circ Res 78: 262-273, 1996.

KHARIN SN: Depolarisation and repolarisation sequences of ventricular epicardium in chickens (Gallus gallus domesticus). Comp Biochem Physiol A Mol Integr Physiol 137: 237-244, 2004.

KHARIN SN, ANTONOVA NA, SHMAKOV DN: Transmural gradient of recovery of excitability of the left ventricle myocardium of the chicken Gallus domesticus. J Evol Biochem Physiol 43: 116-118, 2007.

LAURITA KR, GIROUARD SD, ROSENBAUM DS: Modulation of ventricular repolarization by a premature stimulus. Role of epicardial dispersion of repolarization kinetics demonstrated by optical mapping of the intact guinea pig heart. Circ Res 79: 493-503, 1996.

LI Y, MA J, XIAO JM, LIU N, NIU HY, LU ZY: Heterogeneity of action potential and ion currents in the left ventricular myocytes of the rabbit. Sheng Li Xue Bao 54: 369-374, 2002.

LIBBUS I, ROSENBAUM DS: Transmural action potential changes underlying ventricular electrical remodeling. J Cardiovasc Electrophysiol 14: 394-402, 2003.

LIU G, IDEN JB, KOVITHAVONGS K, GULAMHUSEIN R, DUFF HJ, KAVANAGH KM: In vivo temporal and spatial distribution of depolarization and repolarization and illusive murine T wave. $J$ Physiol Lond 555: 267279, 2003.

MAIN MC, BRYANT SM, HART G: Regional differences in action potential characteristics and membrane currents of guinea-pig left ventricular myocytes. Exp Physiol 83: 747-761, 1998.

MANTRAVADI R, GABRIS B, LIU T, CHOI BR, DE GROAT WC, NG GA, SALAMA G: Autonomic nerve stimulation reverses ventricular repolarization sequence in rabbit hearts. Circ Res 100: e72-e80, 2007.

NISHIMURA M, WATANABE Y, TODA H: The genesis of bifid T waves: experimental demonstration in isolated perfused rabbit hearts. Int J Cardiol 6: 1-16, 1984.

RAMANATHAN C, JIA P, GHANEM R, RYU K, RUDY Y: Activation and repolarization of the normal human heart under complete physiological conditions. Proc Natl Acad Sci USA 103: 6309-6314, 2006.

ROCCHETTI M, FRELI V, PEREGO V, ALTOMARE C, MOSTACCIUOLO G, ZAZA A: Rate dependency of betaadrenergic modulation of repolarizing currents in the guinea-pig ventricle. J Physiol Lond 574: 183-193, 2006.

SICOURI S, ANTZELEVITCH C: A subpopulation of cells with unique electrophysiological properties in the deep subepicardium of the canine ventricle: the M cell. Circ Res 68: 1729-1741, 1991.

SICOURI S, QUIST M, ANTZELEVITCH C: Evidence for the presence of M cells in the guinea pig ventricle. J Cardiovasc Electrophysiol 7: 503-511, 1996.

STANKOVICOVA T, SZILARD M, DE SCHEERDER I, SIPIDO KR: M cells and transmural heterogeneity of action potential configuration in myocytes from the left ventricular wall of the pig heart. Cardiovasc Res 45: 952-960, 2000.

SZABÓ G, SZENTANDRÁSSY N, BÍRÓ T, TÓTH BI, CZIFRA G, MAGYAR J, BÁNYÁSZ T, VARRÓ A, KOVÁCS L, NÁNÁSI PP: Asymmetrical distribution of ion channels in canine and human left-ventricular wall: epicardium versus midmyocardium. Pflugers Arch 450: 307-316, 2005.

SZENTANDRÁSSY N, BÁNYÁSZ T, BÍRÓ T, SZABÓ G, TÓTH BI, MAGYAR J, LAZAR J, VARRÓ A, KOVÁCS L, NÁNÁSI PP: Apico-basal inhomogeneity in distribution of ion channels in canine and human ventricular myocardium. Cardiovasc Res 65: 851-860, 2005.

TAGGART P, SUTTON PM, OPTHOF T, CORONEL R, TRIMLETT R, PUGSLEY W, KALLIS P: Transmural repolarisation in the left ventricle in humans during normoxia and ischemia. Cardiovasc Res 50: 454-462, 2001. 
TATEWAKI T, INAGAKI M, KAWADA T, SHISHIDO T, YANAGIYA Y, TAKAKI H, SATO T, SUGIMACHI M, SUNAGAWA K: Biphasic response of action potential duration to sudden sympathetic stimulation in anesthetized cats. Circ J 67: 876-880, 2003.

TOYOSHIMA H, LUX RL, WYATT RF, BURGESS M, ABILDSKOV JA: Sequences of early and late phases of repolarization on dog ventricular epicardium. J Electrocardiol 14: 143-152, 1981.

TSUJI Y, OPTHOF T, KAMIYA K, YASUI K, LIU W, LU Z, KODAMA I: Pacing-induced heart failure causes a reduction of delayed rectifier potassium currents along with decreases in calcium and transient outward currents in rabbit ventricle. Cardiovasc Res 48: 300-309, 2000.

VOLDERS PG, STENGL M, VAN OPSTAL JM, GERLACH U, SPÄTJENS RL, BEEKMAN JD, SIPIDO KR, VOS MA: Probing the contribution of IKs to canine ventricular repolarization: key role for beta-adrenergic receptor stimulation. Circulation 107: 2753-2760, 2003.

WAN X, BRYANT SM, HART G: The effects of $\left[\mathrm{K}^{+}\right]$o on regional differences in electrical characteristics of ventricular myocytes in guinea-pig. Exp Physiol 85: 769-774, 2000.

WAN X, BRYANT SM, HART G: A topographical study of mechanical and electrical properties of single myocytes isolated from normal guinea-pig ventricular muscle. J Anat 202: 525-536, 2003.

WANG L, FENG ZP, DUFF HJ: Glucocorticoid regulation of cardiac $\mathrm{K}^{+}$currents and L-type $\mathrm{Ca}^{2+}$ current in neonatal mice. Circ Res 85: 168-173, 1999.

WANG L, YONG AC, KILPATRICK D: Effects of procainamide on transmural ventricular repolarisation. Cardiovasc Drugs Ther 16: 335-339, 2002.

XU X, RIALS SJ, WU Y, SALATA JJ, LIU T, BHARUCHA DB, MARINCHAK RA, KOWEY PR: Left ventricular hypertrophy decreases slowly but not rapidly activating delayed rectifier potassium currents of epicardial and endocardial myocytes in rabbits. Circulation 103: 1585-1590, 2001.

YAN GX, MARTIN J: Electrocardiographic T wave: a symbol of transmural dispersion of repolarization in the ventricles. J Cardiovasc Electrophysiol 14: 639-640, 2003.

ZHANG Y-Z, HE B, WANG LX: Effects of an increase in coronary perfusion on transmural ventricular repolarization. Physiol Res 56: 285-290, 2007. 